EPJ Web of Conferences 42, 05005 (2013)

DOI: $10.1051 /$ epjconf/20134205005

(C) Owned by the authors, published by EDP Sciences, 2013

\title{
Improved MOX fuel calculations using new Pu-239, Am-241 and Pu-240 evaluations
}

\author{
G. Noguere ${ }^{1}$, O. Bouland ${ }^{1}$, D. Bernard ${ }^{1}$, P. Leconte ${ }^{1}$, P. Blaise ${ }^{1}$, Y. Peneliau ${ }^{1}$, J.F. Vidal ${ }^{1}$, \\ C. De Saint Jean ${ }^{1}$, L. Leal ${ }^{2}$, P. Schilleebeeckx ${ }^{3}$, S. Kopecky ${ }^{3}$, C. Lampoudis ${ }^{3}$ \\ ${ }^{1}$ CEA, DEN, Cadarache, F-13108 Saint Paul Les Durance, France \\ ${ }^{2}$ Oak Ridge National Laboratory, P.O. Box 2008, Oak Ridge, TN, USA, 37831 \\ ${ }^{3}$ JRC-EC IRMM, Retiseweg, 2440 Geel, Belgium
}

\begin{abstract}
Several studies based on the JEFF-3.1.1 nuclear data library show a systematic overestimation of the critical keff for core configurations of MOX fuel assemblies. The present work investigates possible improvements of the $\mathrm{C} / \mathrm{E}$ results by using new evaluations for Am-241, Pu-239 and Pu-240.
\end{abstract}

\section{Introduction}

Extensive experimental programs, carried out in the zero-power research reactor EOLE of the CEA Cadarache, were designed to study advanced LWR and BWR core configurations of MOX fuel assemblies. Reactivity calculations using the TRIPOLI-4 Monte-Carlo code were reported in many papers. The latest studies, based on the JEFF-3.1.1 nuclear data library, confirm the systematic overestimation of the critical keff. An increasing trend with the Pu ageing is observed. This trend is attributed to the underestimation of the Am-241 neutron capture cross section.

The new evaluation of the Am-241 resonance parameters based on the IRMM and EXFOR data will be shortly presented. Integral results obtained with this new evaluation will be discussed, together with those obtained with the new Pu-239 resonance parameters established in the frame of the working group WPEC/SG-34.

The latest nuclear reaction of interest for this work is the Pu-240 capture cross section. Impact of the radiation width of the broad resonance at $1 \mathrm{eV}$ will be discussed in order to provide recommended values for a future evaluation of $\mathrm{Pu}-240$.

\section{Evaluation of the Am-241 neutron cross sections}

New resonance parameters of the Am-241 cross sections were extracted from recent capture and transmission data measured at the GELINA facility of the IRMM (Geel, Belgium) [1]. The IRMM data were used to extract parameters up to $30 \mathrm{eV}$. Four additional data sets, retrieved from the EXFOR data base, were also included in the Neutron Resonance Shape Analysis. We used the capture yield measured by G. Vanpraet (IRMM, 1985) and the transmission data measured by H. Derrien (Saclay, 1975). These data allowed the analysis of the resolved resonance range up to 150 
eV. For the fission cross section, the data measured by Derrien (Saclay, 1975) and Dabbs (ORELA, 1983) were analyzed with the CONRAD code. Results are shown in Fig. 1.
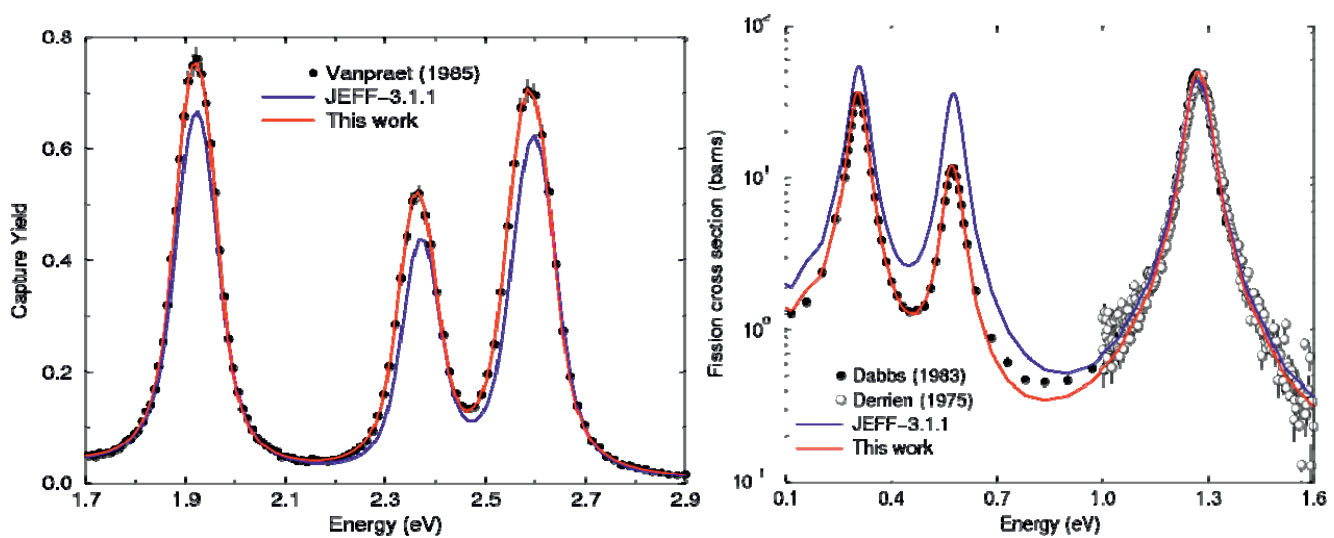

Fig. 1. Am-241 capture yield and fission cross sections obtained in this work.

Above the resolved resonance range, the cross sections of the neutron induced reactions were calculated with the ECIS and TALYS codes. The optical model calculations were performed by using the optical model parameters reported in JENDL-4 for the nuclear system $n+A m-241$. The fission cross section was determined in collaboration with the University of Bucarest in the frame of the PhD thesis of Claudia Morariu. The sequences of "head band states" needed to describe the fission process were established by using methodologies developed at LANL by E. Lynn [2].

The results were converted in ENDF-6 format and tested with a series of integral benchmarks performed at the MELUSINE, EOLE, MASURCA, PHENIX and MINERVE reactors. Calculations were performed with the APOLLO-2.8, TRIPLI-4 and ERANOS-2 codes. For the thermal capture cross section and for the capture resonance integral, the ICARE and SHERWOOD experiments, performed at the MELUSINE reactor of Grenoble, confirm the value of $\sigma_{\gamma}=750$ barns and $\mathrm{I}_{0}=1825$ barns given by the IRMM data.

\section{Evaluation of the resonance parameters of Pu-239}

The Pu-239 evaluation of JEFF-3.1.1 was produced with an obsolete option of the ENDF-6 format. As a consequence, a new evaluation of the resolved resonance range was performed in the frame of the working group WPEC/SG-34. Detailed descriptions of the evaluation work will be given in the final report planed in 2013.

The new set of resonance parameters was established by H. Derrien at ORNL. The parameters of the lower energy range were improved at the CEA Cadarache in collaboration with L. Leal to account for integral benchmarks [3]. Final results are presented in Fig. 2. The phenomenological description of the fluctuations of $v_{p}$ was also investigated by introducing the contribution of the (n, $\left.\gamma \mathrm{f}\right)$ process. As shown in Fig. 3, the present work confirms the description available in JEFF-3.1.1.

The Resonance Parameters Covariance Matrix (RPCM) was established with the CONRAD code by using a marginalization procedure. The principle of the retroactive analysis was applied up to $1 \mathrm{MeV}$ in order to produce covariance information without changing the magnitude of the cross sections. The results are shown in Fig. 4. 


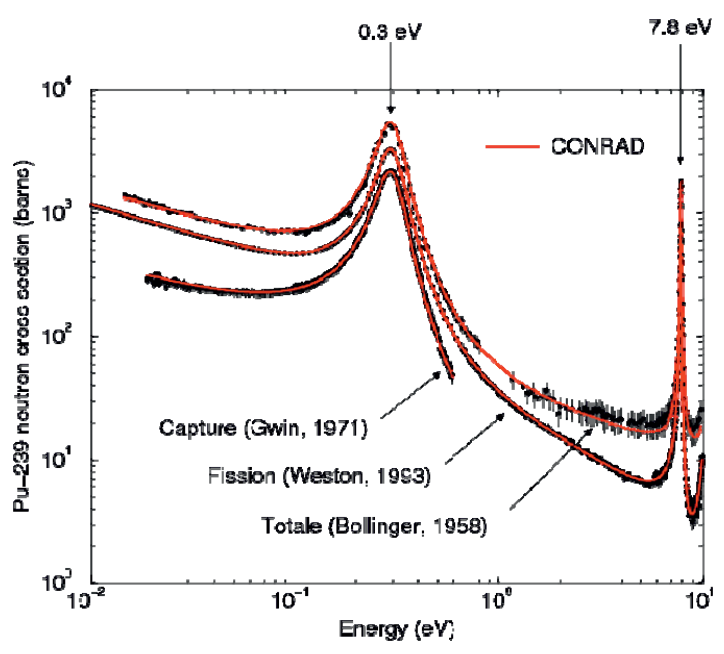

Fig. 2. Pu-239 Cross section calculated with the CONRAD code.

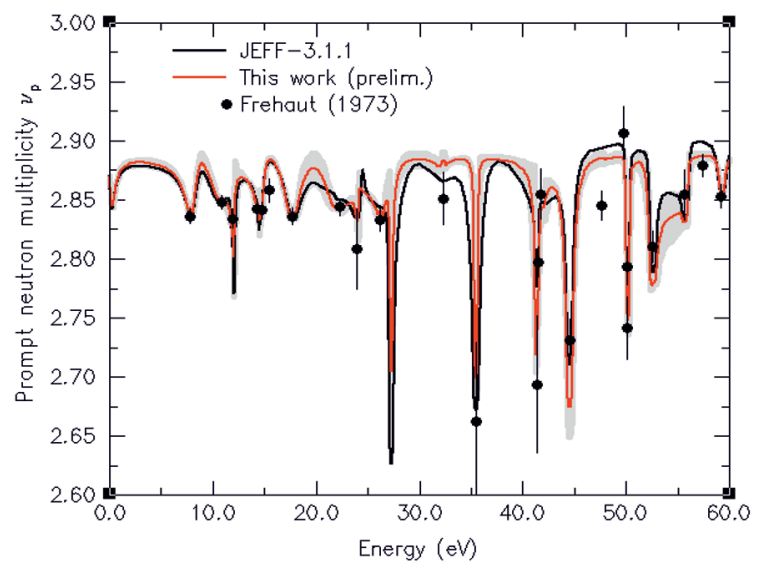

Fig. 3. Pu-239 prompt neutron multiplicity obtained in this work.

\section{Sensitivity studies of the radiation width of $\mathrm{Pu}-240$ at $1 \mathrm{eV}$}

For the latest isotope of interest for this work, we have investigated the impact of the radiation width of the first broad resonance at $1 \mathrm{eV}$. This sensitivity study was motivated by unpublished results of A. Meister. A comparison of the parameters of the first resonance obtained with the Free Gas Model (FGM) and the Cristal Lattice Model (CLM) indicate that the radiation width can be underestimated by at least $1 \mathrm{meV}$ if the crystal lattice effects are not taken into account. Results are summarized in Table 1. 


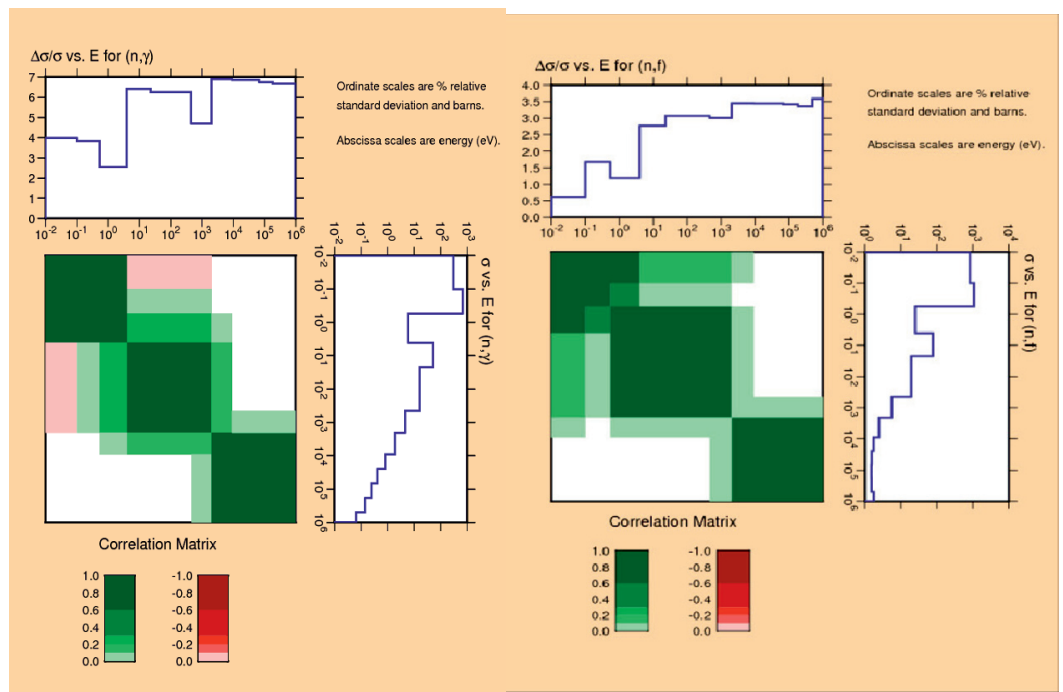

Fig. 4. Covariance matrix for the Pu-239 cross sections calculated with CONRAD up to $1 \mathrm{MeV}$.

The impact of the uncertainty on the radiation width was estimated with ICSBEP benchmarks characterised by a high content of $\mathrm{Pu}-240$. For this work we have selected a single critical benchmark PST018.6 (43\% of Pu-240). A series of TRIPOLI-4 calculations indicate that an increase of $1 \%$ of the radiation width leads to a significant decrease of $-230 \pm 50 \mathrm{pcm}$ of the calculated reactivity. Similar trends are obtained for the MISTRAL-2 and MISTRAL-3 experiments performed in the EOLE facility of the CEA Cadarache.

Table 1. Values of the Pu-240 radiation width of the first broad resonance at $1 \mathrm{eV}$.

\begin{tabular}{|c|c|c|c|}
\hline \multirow{2}{*}{ Doppler Model } & Author & Reference & Radiation width \\
\hline \multirow{2}{*}{ FGM } & O. Bouland & NSE 127, 105 (1997) & $29.14 \pm 0.6 \mathrm{meV}(2 \%)$ \\
\cline { 2 - 4 } & S.F. Mughabghab & Atlas of Neutron Resonances, 2006 & $29.14 \pm 0.6 \mathrm{meV}(2 \%)$ \\
\cline { 2 - 4 } & JEFF-3.1.1 & & $29.15 \mathrm{meV}$ \\
\hline \multirow{2}{*}{ CLM } & A. Meister & Private communication & $30.50 \pm 0.31 \mathrm{meV}(1 \%)$ \\
& & & $30.39 \pm 0.13 \mathrm{meV}(0.4 \%)$ \\
\hline
\end{tabular}

\section{Integral Results}

Among the extensive experimental database available at the CEA Cadarache, we have selected LWR-MOX cores of the MISTRAL program (MISTRAL-2, MISTRAL-3, MISTRAL-4), UOXMOX mixed cores of the EPICURE program (MH1.2) and BWR-MOX cores of the FUBILA program. Results obtained in this work are shown in Fig. 5. 


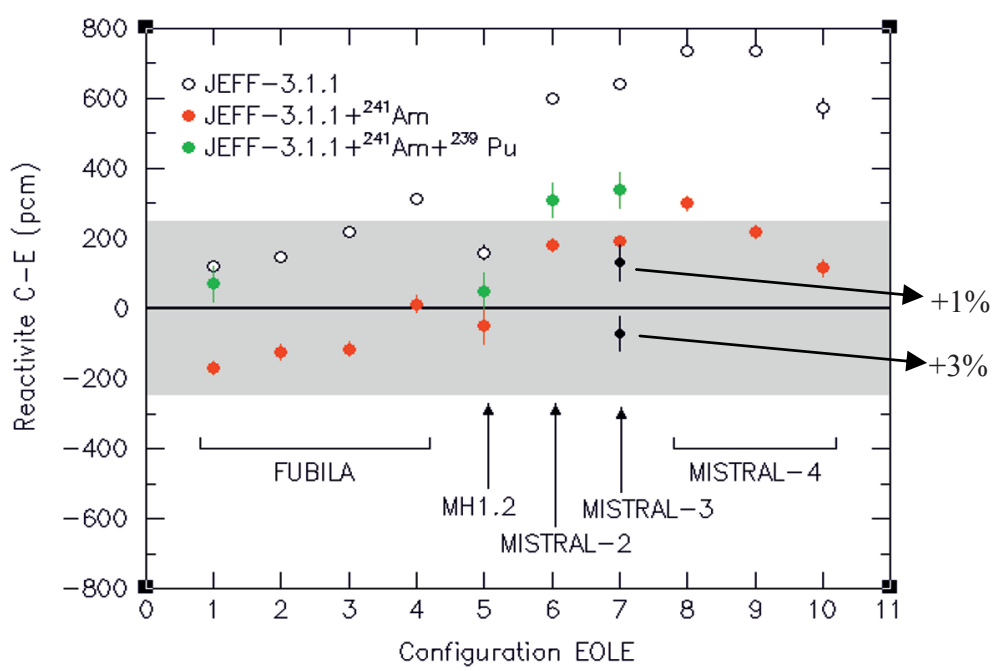

Fig. 5. Integral results obtained for several experiments performed in the EOLE facility of CEA Cadarache.

The new evaluation of Am-241 performed in collaboration with IRMM improves significantly the $\mathrm{C} / \mathrm{E}$ results for the EOLE experiments. However, a systematic discrepancy seems to exist between the FUBILA and MISTRAL results. The new evaluation of $\mathrm{Pu}-239$ tends to reduce the observed discrepancy. Additional TRIPOLI-4 calculations confirm the impact of the radiation width of the first resonance of $\mathrm{Pu}-240$. Results obtained from a direct perturbation of $1 \%$ and $3 \%$ are reported on Fig. 5 for the reference configuration of the MISTRAL-3 program. These latest results confirm the needs for new Pu-240 measurements at low temperature in order to analyze the resonances with an appropriate phonon spectrum.

\section{Conclusions}

The work reported in this paper demonstrates the performances of the new Am-241 evaluation based on capture and transmission data measured at the IRMM. For Pu-239, the new evaluation, established in the frame of the WPEC/SG-34, is able to explain a systematic discrepancy observed between different EOLE experiments. The combination of the Am-241 and Pu-239 evaluations demonstrates the necessity to improve the radiation width of the first resonance of $\mathrm{Pu}-240$.

\section{References}

1. C. Lampoudis et al., Neutron transmission and capture of Am-241 from thermal up to $100 \mathrm{eV}$, this workshop.

2. O. Bouland et al., Intermediate structure in fission - Consequences on average partial cross sections, this workshop.

3. A. C. (Skip) Kahler et al., Critical eigenvalue calculations of selected ICSBEP benchmarks with various $\mathrm{Pu}-239$ evaluated data files, this workshop. 